\title{
PSF correction in soft x-ray tomography
}

\author{
Axel Ekman ${ }^{a}$, Venera Weinhardt ${ }^{\mathrm{a}}$, Jian-Hua Chen ${ }^{\mathrm{a}}$, Gerry McDermott ${ }^{\mathrm{a}}$, Mark A. Le Gros ${ }^{\mathrm{a}, \mathrm{b}}$, \\ and Carolyn Larabella,b, ${ }^{*}$ \\ aDepartment of Anatomy, University of California, San Francisco, CA 94143, USA \\ bMolecular Biophysics and Integrated Bioimaging, Lawrence Berkeley National Laboratory, \\ Berkeley, CA 94720, USA
}

\begin{abstract}
In this manuscript, we introduce a linear approximation of the forward model of soft x-ray tomography (SXT), such that the reconstruction is solvable by standard iterative schemsses. This linear model takes into account the three-dimensional point spread function (PSF) of the optical system, which consequently enhances the reconstruction data. The feasibility of the model is demonstrated on both simulated and experimental data, based on theoretically estimated and experimentally measured PSFs.
\end{abstract}

\section{Keywords}

3D imaging; image processing; tomography

\section{Introduction}

Soft $\mathrm{x}$-ray tomography (SXT) refers to the x-ray microscopy technique in which tomographic imaging is done using low-energy $\mathrm{x}$-rays. In particular, the $\mathrm{x}$-ray energy range lies within the "water window", i.e., between the K-absorption edges of oxygen $(2.34 \mathrm{~nm}$; $530 \mathrm{eV})$ and carbon $(4.4 \mathrm{~nm} ; 280 \mathrm{eV})$ [1]. As the name suggests, water is relatively transparent to the x-rays within this region. In biological samples, contrast arises from the natural variation of bio-organic molecules making this region especially suitable for imaging of these kinds of samples. In the past decades, soft x-ray microscopy has emerged as a unique tool to study $3 \mathrm{D}$ organization of single cells $[4,8,33]$. From simple yeast to complex eukaryotic cells. SXT grants an unprecedented contrast and spatial resolution, filling the information gap between light and electron microscopy [43, 37, 13, 6, 21, 5].

The three-dimensional reconstruction of a sample is obtained, by solving for its spatial distribution of absorption from a series of projection from many different viewing angles around a central rotation axis. A key assumption has traditionally been that these images can be regarded as images of classical projections [48], meaning that the resolution would be limited by that of the optical system, $r a \lambda / N A$, where $\lambda$ is the wavelength of the illuminating light and $N A$ is the numerical aperture of the objective lens. Diffraction limited

\footnotetext{
*Corresponding author carolyn.larabell@uscf.edu (Carolyn Larabell).
} 
optics are, however, also characterized by a maximum depth of field as DOF $a \lambda / N A^{2}$, introducing a limit of sample size where the image formation can be approximated by this kind of parallel projection [28], and where the effect of the optics can be modeled by a depth independent point spread function (PSF).

In principle, both a sufficient depth of field and a good resolution can be obtained by increasing the energy (decreasing $\lambda$ ) and decreasing the $N A$ (see Fig. 1). However, natural contrast in bio-organic samples is limited to the energy region of the water window, making such optimization impossible. This means that, especially for larger samples, SXT resolution suffers from the depth-dependent optical PSF.

Limited DOF is a known problem in electron microscopy, where tilting of the sample may extend part of the sample out of focus, and approximate solutions exist to correct for it in the tomographic reconstruction. The so-called defocus-gradient correction, which was first introduced by Jensen and Kornberg [15], involves computational correction of the effects of a depth-dependent defocus and was incorporated to the well-known back-projection algorithm. The problem was revisited by Kazantsev et al. [17], in which the method received rigorous mathematical justification. This kind of correction has also been applied in Fourier space $[47,46]$, which can reduce the computational times up to two orders of magnitude. However, these corrections are not directly applicable in SXT due to the difference in image formation [20].

In SXT the problem of limited DOF has only recently been addressed, and thus far only experimentally. This has been done by acquiring multiple images at different focus and using a depth-dependent weight on their backprojection to account for their different foci [39], by using through-focus imaging to then computationally extract the ideal projection [26] or by a wavelet based fusion of reconstructions with different foci [23].

Recently, a forward model of image formation in SXT was proposed [28, 29], including rigorous mathematical work $[19,18]$. Based on this model, the feasibility and practical application of PSF corrections was shown by Otón et al. [27], where a depth independent correction was applied to SXT data, leading to higher contrast in the obtained reconstruction.

In this manuscript we further generalize the image formation in soft x-ray tomography developed by Otón et al. [28]. By applying a linear approximation on the model, the effects of a depth dependent PSF can be incorporated into existing iterative reconstruction methods. We present numerical results that show the method is applicable even if the sample is out of focus, or larger than the depth of field. Finally, experimental results show an increase in contrast of a reconstructed image of mouse lymphocytes.

\section{Image formation in soft x-ray tomography}

The understanding of image formation is an important step in any imaging techniques. It allows to choose the best image acquisition strategies and most suitable reconstruction methods. In tomography, the model of image formation has been traditionally based on the Radon transform [35], which is the ideal linear transform (projection) of the specimens' 
attenuation coefficients onto a plane. The projection is linked to the experimental image formation through the Beer-Lambert law, such that the attenuation of the intensity along the ray-paths, $L_{i}$, through the sample can be given by

$$
\int_{L_{i}} \mu_{L_{i}}(t) \mathrm{d} t=-\ln \left(\frac{I_{i}}{I_{i 0}}\right)
$$

Here $\mu L_{i}(t)$ is the linear absorption coefficient (LAC) of the specimen along the ray-path, and $I_{i}$ and $I_{i 0}$ are the attenuated and un-attenuated intensities along the ray-path, respectively.

Although this is a convenient model and a good approximation for highly elongated point spread functions, the image formation in x-ray tomography based on, e.g., diffraction lenses may differ substantially from such ideal model of parallel projections. Despite of this difference, it is beneficial to link the model of image formation in SXT to Eq. (1), so that many available reconstruction methods $[25,16,12]$ become available.

Recently, the specifics of the optical system in SXT were integrated into a model of image formation $[28,29]$. The model assumes, that propagation of the field, at the vicinity of the sample, can be done by parallel propagation, which is valid if the $N A$ of the system is small enough. On the other hand, the model assumes incoherent image formation on the detector, which is reasonable if the $N A$ of the condenser and objective lens are matched, so that the image $I_{\mathrm{im}}$ formed at the detector plane $x_{2}=\left(x_{2}, y_{2}\right)$ is given by

$$
I_{\mathrm{im}}\left(x_{2}\right)-I_{\mathrm{im}}^{0}\left(x_{2}\right)=\int_{R}\left(-\mu\left(x_{1}, z\right) I\left(x_{1}, z_{0}\right) e^{\int_{z_{0}}^{z}-\mu\left(x_{1}, t\right) \mathrm{d} t}\right) * *\left|h_{z}\left(x_{1}\right)\right|^{2} \mathrm{~d} z
$$

where $I\left(x_{1}, z_{0}\right)$ is the local field intensity at some position $z_{0}$ before the sample, $* *: \mathbf{R}^{2} \rightarrow$ $\mathbf{R}^{2}$ is the convolution operator in two dimensions, and $h_{Z}\left(x_{1}\right)$ is the impulse response of the optical system. $I_{\mathrm{im}}\left(x_{2}\right)$ and $I_{i m}^{0}\left(x_{2}\right)$ denotes the recorded images, the latter a reference image without a sample (see Fig. A.8 for details).

Otón et al. [28] describes two cases in which a known solution exist. In one case, when the impulse response $h_{Z}\left(x_{1}\right)$ is a delta function, the model coincides with Eq. (1). In the other case, the impulse response $h_{Z}\left(x_{1}\right)$ is independent of the axial position $z$. As a result, the ideal Beer-Lambert projections can be recovered by deconvolution of the transmission images, after which conventional reconstruction schemes can be applied.

For a more general solution, in our work, we seek a linear approximation to the forward model Eq. (2), so the image formation in SXT can be expressed as

$$
-\ln \frac{I_{\mathrm{im}}}{I_{\mathrm{im}}^{0}} \approx A_{h} \mu
$$


where $\boldsymbol{A}_{\boldsymbol{h}}$ is a linear projection matrix incorporating the PSF of the system and $\boldsymbol{\mu}$ is a discretized vector representation of the LAC.

By following the steps of Otón et al. [28] and building up the left-hand side of Eq. (3). The finite difference (see Appendix for details), yields a linear approximation of the form

$$
A_{h} \mu=\int_{\mathrm{R}} \mu(x, z) * * h_{z}(x)^{2} \mathrm{~d} z
$$

where $\boldsymbol{A}_{h}$ is a projection matrix but now incorporating the depth dependent PSF.

\subsection{Partial coherence}

In practice, this linear approximation Eq. (4) is always associated with error, depending on the resolution and spatial coherence length of the illumination. As partially coherent imaging is described by a bilinear transformation of the complex field [14], these effects cannot fully be captured by a linear projection matrix $\boldsymbol{A}_{h}$. Indeed, x-ray microscopy shows in both simulations [38] and measurements [30, 24] noticeable deviations from a pure incoherent image formation.

The applicability of our model to $\mathrm{x}$-ray microscopes with partial coherence depends on the validity of such a linear approximation describing complex field. For weak objects where interference between diffracted light can be neglected, the image formation becomes linear and can be described by a weak-object transfer function (WOTF) [42, 40]. This is particularly suitable for soft $\mathrm{x}$-ray illumination, as the scattering cross section for bioorganic molecules is typically over three orders of magnitude smaller than that of absorption [11] and thus can be neglected. As the WOTF depends on the partial coherence of the illumination, within linear accuracy it can be incorporated in the projection matrix, $\boldsymbol{A}_{h}$. Other effects not explained by incoherent image formation [24] and cases where weakobject approximation is not valid are still taken into account in the reconstruction with the best accuracy possible in case of projection (intensity-based) imaging.

In our work, we base our model on the inchoherent assumptions as presented in [28]. An indepth investigation of the limits of the linear approximation as applied to realistic microscope parameters, including numerical modeling of the image formation [45, 2, 38], is beyond the scope of this article.

\section{Numerical results}

To validate our, now linear, image formation model, we first performed numerical simulations on a phantom sample, Fig. 2, based on an annotated human B cell. For simplicity the LAC values of organelles were scaled from 0 to 1 . Projection images through the phantom sample were calculated using the non-linear model of (Eq. 2$)$ on a larger ( $L=$ $997^{1}$ ) discrete grid. The sinograms were down-sampled to size $L=256$ before tomographic

\footnotetext{
${ }^{1}$ All lengths describing the numerical validation, are in units of pixels.
} 
reconstructions. To avoid inversion crimes, the size of high-resolution grid was chosen as a prime number, so that neither the reconstruction or deconvolution was done in the same discrete grid as the calculated phantom.

The theoretical PSF was calculated according to the dimensions of the reconstruction grid and was determined as in Ref. [48], by the converging illumination emerging from a circular lens aperture based on the Huygens-Fresnel principle [3]. The LAC of the phantom was scaled so that the minimum transmission was $0.5 I_{0}$.

As a proof of concept, two PSFs were considered. For a low-resolution case, we chose a PSF with a DOF of \pm 128 , i.e., providing a rather elongated PSF enclosing the whole sample (256 pixels wide), and a Rayleigh resolution of 8 units. The second PSF used, mimicked an increase in $N A$, such that the Rayleigh resolution is halved (4 pixels). Correspondingly the DOF of this PSF is thus \pm 32 and spread over a quarter of the cell only. For this high resolution PSF the focal spot was shifted slightly towards the edge of the image to induce axial asymmetry. The sampling criteria of $n>L(\pi / 4)$ was used for reference and the reconstructions were made using 201 projections $^{2}$. The final projections were distorted by adding Poisson noise.

Three different reconstructions (as shown in Fig. 3) were obtained by solving (Eq. 3) by using the Conjugate Gradient Method on the Normal Equations (CGNE): a conventional minimization using the Beer-Lambert approximation, the depth-independent correction of [28], and the linear PSF model.

In CGNE, the iteration number can be viewed as a regularization parameter for the solution [10] and running too many iterations will result in amplifying high frequency signals and result in noisy reconstructions. The optimal stopping iterations will of course be data dependent. So as to ensure a fair comparison of the phantom images, we used the oracle knowledge of the phantom to find the highest peak-signal-to-noise-ratio (PSNR)

$$
\operatorname{PSNR}\left(x, x_{\text {ref }}\right)=20 \log _{10}\left[\frac{\max \left(x_{\text {ref }}\right)-\min \left(x_{\text {ref }}\right)}{\left\langle\left(x-x_{\text {ref }}\right)^{2}\right\rangle}\right]
$$

This was done by keeping track of the best solution within the reconstruction scheme and halting when no improvement over the best solution had been recorded within 5 iterations. For the reconstructions shown in Fig. 3 with $I_{0}=10^{6}$ the stopping iteration numbers for the Beer-Lambert, deconvolution and PSF reconstructions, respectively were 17,19, and 146 iterations for the low resolution case, and 22, 24, and 102 iterations for the high resolution PSF (the corresponding curves of PSNR as a function of stopping iteration are shown in supplementary material, Fig. S.1).

\footnotetext{
${ }^{2}$ The odd number of projections is convenient, as it ensures that the same sampling could be used for both 180 and $360^{\circ}$ If $n$ is even and the sampling is done over $360^{\circ}$, the angles will be at a $\pi$ shift and do not provide any additional sampling if the PSF is in focus and symmetric with respect to the defocus.
} 
The optimal deconvolution for the depth-independent correction of [28] was done in two steps - first, noiseless Beer-Lambert projection images were as a convolution kernel to solve for the optimal depth-independent PSF. This 2D kernel was then used to deconvolve the projection images, using the oracle knowledge of the same Beer-Lambert projections as stopping criteria by minimizing the $l_{2}$-norm of the residual.

As seen from the reconstructions in Fig. 3, for a centered PSF, the result is as expected. The Beer-Lambert approximation shows smoothing of the edges, characteristic to the PSF. In this case, as the assumption of a depth independent PSF is valid, a proper solution to the inversion exist [28] and global filtering of the projection images yields a good reconstruction result. The linear PSF inversion is similar in quality, but in the case of centered PSF, the deconvolution-based reconstructions have the benefit of much faster convergence. As seen from the large area of constant LAC, the optimal PSF inversion also shows slightly more ringing, than the deconvolved example indicating that the inversion could benefit from a suitable regularization term.

In the asymmetric high-resolution case, the Beer-Lambert reconstruction clearly shows the artifacts of the defocus. In this case, no suitable global filter exists, and the reconstructions based on projection image deconvolution suffers from the same kind of artifacts. Although effects of defocus are visible near the lower-left edge of the phantom, both depthindependent and linear PSF reconstructions improve the quality of the data, improving on the quality of the reconstruction and relative contrast between organelles. The linear PSF reconstructions are significantly better handling the asymmetry of the PSF as shown in the significant increase in PSNR in the lower left panel of Fig. 3.

In numerical simulation, where the projection matrix corresponding to the PSF is known, the linear approximation performs well for the tomographic inversion. In practice, however, the image quality is often limited by noise, which makes the inversion problem highly unstable. To investigate the stability of the inversion, the numerical experiment was repeated using different noise levels. We show in Fig. 3 the PSNR of the recovered image as a function of the intensity count. As all images were distorted with Poisson noise, a lower count corresponds to a higher noise level. For all cases, the linear PSF inversion provides the highest PSNR and is most resilient to the effects of this noise. For methods when the model of image formation is insufficient, the quality seems to saturate as image quality increases, which can be seen from the high intensity PSNR for the Beer-Lambert reconstructions. In this regime, the image model error dominates over the error caused by the noise in the images.

For an out of focus PSF, as the one used for the high-resolution case, one can stabilize the reconstructions by taking images over a full $360^{\circ}$ rotation range. In this fashion, though a sample is not fully in focus at one rotation angle, the sampling at a $180^{\circ}$ shift provides additional information, as a different part of the sample will be in focus. Such acquisition can be seen as a modification to the method suggested by Selin et al. [39], where a depthdependent weight was introduced in the reconstruction scheme to account for projections acquired at different foci. In the presented method, the projection matrix $\boldsymbol{A}_{h}$ servers a similar function. Such acquisition scheme leads to better reconstruction results in comparison to the 
$180^{\circ}$ rotation, using the same number of projections, with all methods (Reconstructions shown in Supplementary Materials). The full set of reconstructions used for Fig. 3 are shown in the supplementary material, Figs S2 to S5.

To investigate the limits of the linear PSF model, we conducted a series of reconstructions where the resolution was kept constant but the DOF was decreased with respect to the sample size (see Fig. 4). The reduced DOF results in radial blurring, that is loss of resolution and contrast between organelles within cell. When DOF becomes approximately half of the region of interest (ROI), this effect becomes severe. Both depth-independent and linear PSF reconstructions allow to amend these effects. Though the linear PSF model results in radial artifacts for extremely small DOF ( $23 \%$ of the phantom), its improvement is superior with respect to depth-independent schemes.

\section{Experimental results}

To test the method on experimental data, the PSF of the optical system must first be measured. This PSF can then be used to calculate the necessary weights for the projection (and back-projection) operators used in the reconstruction. The experimental work was performed at the National Center for X-ray Tomography at the Advanced Light Source of the Lawrence Berkeley National Laboratory. The data was acquired on the XM-2 soft x-ray microscope [22]. The XM-2 is equipped with a cryogenic rotation stage with full $360^{\circ}$ range to enable tomographic data collection from cryo-preserved samples. The optical setup consists of two aperture matched Fresnel Zone Plates, with a relatively low NA (0.234), thus the assumptions needed for Eq. (2) should be met. However, although the theoretical framework rests on the assumption of incoherent image formation, it puts no restrictions into the actual linear operator $\boldsymbol{A}_{h}$ and when measuring the PSF, it is straightforward to incorporate also other effects in the projection matrix, such as various aberrations, distortions, or a spatially varying PSF.

\subsection{Measuring the system PSF}

To measure PSF of the soft x-ray microscope optics, we prepared the phantom sample composed of gold nanoparticles. Spherical gold nanoparticles (Nanopartz, Cat.No. AR11100 -NB-50) with diameter of $(100 \pm 4) \mathrm{nm}$ were deposited with a microloader on a $100 \mathrm{~nm}$ thick silicon nitride membrane (Silson LtD, Ref.10402101) and then spread by a gentle flow of warm air. The distribution of nanoparticles was confirmed by optical microscopy in dark field mode. The areas with single isolated particles were selected for imaging with x-ray microscope. The regions of interest were imaged with a set of 30 through-focus images with $1 \mu \mathrm{m}$ step size and $150 \mathrm{~ms}$ exposure time each. For each set of radiographs, 20 reference images were acquired with the same exposure time. The radiographs were recorded by a charge-coupled-device camera (Andor IKon-L) with an effective pixel size of $16 \mathrm{~nm}$. A reference profile was obtained by fitting the theoretical intensity function,

$$
p(x)=I_{0} \exp \left[-2 \mu \sqrt{R^{2}-\left(x-x_{c}\right)^{2}}\right],
$$


to the experimental data of the in-focus image. Here, $I_{0}$ is the initial intensity, $\mu$ is scaled LAC, and $x_{\mathcal{C}}$ is center of a sphere.

The PSF was determined by maximum-likelihood (ML) deconvolution [44, 49]. Essentially, we assume that the image of a single bead can be written in form $\boldsymbol{y}=\boldsymbol{p} * * \boldsymbol{h}+\boldsymbol{\epsilon}$, where the measured signal $\boldsymbol{y}$ is composed of a convolution between the bead profile, $\boldsymbol{p}$, the PSF, $\boldsymbol{h}$, and an additional noise term $\epsilon$. The ML solution for the PSF can now be found iteratively, using the fitted theoretical bead profile Eq. (6), with the Richardson-Lucy algorithm:

$$
\boldsymbol{h}_{k+1}=\boldsymbol{h}_{k}\left[\frac{\boldsymbol{y}}{\boldsymbol{p}^{*} * \boldsymbol{h}_{k}} * \hat{\boldsymbol{p}}\right]
$$

where $\hat{\boldsymbol{p}}$ is the adjoint of $\boldsymbol{p}$. A volumetric representation of the PSF was obtained by bilinear interpolation into a discrete grid, an example of a PSF extracted in this way is shown in Fig. 5, from which transverse slices give the needed 2D convolution kernels, $\boldsymbol{h}_{Z}$ for (Eq. 4). The final PSF was taken as an average over five separate extracted PSFs. The full-width-at-halfmaximum of separately fitted gaussian distributions to the in focus PSFs correspond to (59 $\pm 3) \mathrm{nm}$, and a maximum loss of intensity of $20 \%$ [3, p. 441] along the axial direction gives a DOF of $\pm(4.7 \pm 0.2) \mu \mathrm{m}$. To check whether the inversion was stable within measured error bounds, numerical simulations were performed using mismatching PSFs for the forward and backward model. Substantial artifacts arise from two cases: A substantially wider PSF or a substantially smaller DOF. Reducing the resolution of the PSF will eventually converge with the Beer-Lambert model, thus the method is rather robust against underestimating the resolution. As shown in Supplementary materials (Figs S.6 and S.7) substantially larger perturbations were needed for notable deviations in image quality for a PSF of similar elongated shape, thus the measured PSF is sufficiently accurate to provide a projection operator for the inversion.

\section{4-2. Experimental validation: imaging of mouse B cell}

To test the applicability of the method for experimental data, the method was used to reconstruct tomographic images of mouse lymphocytes. To have full angle rotation during the acquisition, the cells were loaded into thin-wall glass capillaries with a micro loader [31]. For cryo-fixation, the capillaries were rapidly plunged into a liquid propane cooled by liquid $\mathrm{N}_{2}$. The data acquisition was done by sequentially rotating the capillary with $2^{\circ}$ increments for $180^{\circ}$ with an exposure time of $300 \mathrm{~ms}$ per image. A series of 10 reference images were taken before and after the scan, that were used to normalize the data. The projection images were aligned using an updated version of the previously developed automatic registration software AREC3D [32].

The tomographic image was reconstructed in three different ways using CGNE, viz.: A reference image using the Beer-Lambert approximation, a reconstruction where the intensity images are deconvolved with a 2D PSF, i.e., the solution to Eq. (2) if the PSF is not depthdependent, and using the linear PSF approximation presented in this paper. The 2D kernel for the deconvolution was obtained by taking the average of all the measured 2D kernels 
within the DOF of the PSF. The deconvolution was performed on the transmission images using RL deconvolution and stopped before significant ringing was observed ${ }^{3}$.

The reconstructed, based on linear PSF, virtual slices through a 3D volume of a mouse B cell are shown in Fig. 6. For comparison the same slices reconstructed with Beer-Lambert and depth-independent deconvolution are shown in supplementary material (Fig. S.8). We have selected two regions with endoplasmic reticulum for the comparison of three different reconstruction schemes. As the PSF of the microscope (see Fig. 5) is larger than thickness of the specimen, there is no noticeable DOF effects. In terms of contrast between the organelles, both PSF corrected reconstructions show an increase of contrast with respect to the Beer-Lambert approximation. The transverse slice (plane $x y$ ) show an increase of contrast between endoplasmic reticulum and cytoplasm of $10 \%$ to $20 \%$. The effect is less pronounced in the in-plane (plane $z X$ ) reconstruction ( $0 \%$ to $10 \%$ ), where image quality is limited by the relatively sparse sampling.

\subsection{Experimental validation: segmentation of a human $\mathrm{T}$ cell}

While the difference between reconstruction schemes in terms of contrast is rather moderate, it can provide great advantage for segmentation and annotation of data. Particularly for automatic segmentation $[36,7,41,50,34]$ this increase in contrast might be the key to successful segmentation.

As a test for this, we used SlideCAM ${ }^{4}$ environment to apply the algorithm presented by Pelt and Sethian [34] on a SXT image of a human T cell, reconstructed both with a Beer-Lambert approximation and the linear PSF approximation. The convolutional neural network (CNN) was trained on a transverse (plane $x z$ ) and tested on the plane orthogonal of the trained data set (plane $y z$ ). The weight of the endoplasmic reticulum (ER) was not large enough to have a noticeable effect on the global loss function, so it was classified with a separate CNN, trained in the same manner.

An example slice of the classified reconstruction is shown in Fig. 7. The corresponding precision (the fraction of the predicted pixels that were correct) and recall (the fraction of the labeled pixels that were predicted) for each of the classification labels are tabulated in Table 1. As expected, the overall precision of the classification is similar, 0.921 for the BeerLambert reconstruction and 0.927 for the PSF model. In terms of the larger features in the image, viz: identifying the cell, and its nucleus and nucleoli, the reconstructions produced similar results. The PSF reconstruction showed better precision in recognizing mitochondria, but was more selective, resulting in a lower recall of the classification. The difference in the reconstruction is most notable in enhancing the detectability of sharp features, such as membranes or ER, which can be seen by a 1.7 fold increase in precision and 5.4 fold increase in the recall of the classification of ER.

\footnotetext{
${ }^{3}$ Of course, as the experimental data lacks oracle information this could not be done in as rigorous manner as for the phantom sample, so the "best" result had to be qualitatively determined by visual inspection.

4 https://slidecam-camera.lbl.gov/
} 


\section{Conclusions}

In this work we have introduced a linear approximation to the reconstruction of tomographic $\mathrm{X}$-ray data, including the effect of the point spread function of the optics. We show numerically, that the approximation is well suited for the inversion of the SXT data both when the PSF acts as depth-independent blur as well as when the sample is partially out of focus.

For experimental measurements, the PSF inversion based on a linear approximation increases contrast in the image, especially of edge features, which show a relative enhancement up to $20 \%$. The method provides similar results as compared to the non-linear deconvolution approach. Numerical results show that the introduced inversion using a projection matrix including the PSF is more resilient to noise than its deconvolution-based counterpart, most likely since it is directly applied on the $3 \mathrm{D}$ volume and not on the projections, where the PSF inversion is done independently. This, however, comes at a price, since the reconstruction using the PSF incorporated projection matrix is both computationally more expensive, and converges significantly slower.

On one side, linear PSF reconstructions provide better relative contrast in the cells, guaranteeing correct annotation of organelles and easier, mainly automatic segmentation. On the other side, without the need for imaging schemes it helps to increase contrast and spatial resolution achievable with SXT for thicker cells.

The presented model tackles current limitations of SXT and provides new ways of data acquisition. For a properly sampled and focused PSF, the linear PSF approximation enables use of higher $N A$ optics, as the limited depth of field can be computationally amended. However, further studies may be needed to address the issue of partial coherence as the objective $N A$ becomes larger.

The results in Fig. 3 predict a possible way to extend the depth of field experimentally by shifting the focus to either side of the sample and taking a full $360^{\circ}$ rotation data set. This would allow one to circumvent the traditional limitations of diffraction limited optics Fig. 1 and extend the effective depth of field with respect to the resolution. In other words, it would either enable imaging current samples with a higher $N A$, thus a higher resolution, or extending the DOF of current imaging systems, allowing for full 3D reconstructions of larger samples.

We expect the method to be generally applicable also for other tomographic systems, if the assumption of a linear image formation can be met.

\section{Supplementary Material}

Refer to Web version on PubMed Central for supplementary material.

\section{Acknowledgment}

Research reported in this publication was conducted at The National Center for X-ray Tomography, located at the Advanced Light Source of Lawrence Berkeley National Laboratory, and was supported by grants from NIH 
(P41GM103445), DOE's Office of Biological and Environmental Research (DE-AC02-5CH11231), and Chan Zuckerberg Initiative Human Cell Atlas program. Venera Weinhardt was funded by German Research Foundation fellowship WE6221/1-1.

\section{Appendix A.: Linear approximation of the image formation}

The model of Otón et al. [28] is based on two assumptions, in which the measured projection is a result of the attenuated light passing through the sample, blurred by the PSF of the objective lens. We assume firstly, that there exists an impulse response function $h_{Z}\left(x_{1}\right.$, $x_{2}$ ) (see e.g. Refs [3] and [9] for details), such that the intensity field at the image plane, $I_{\mathrm{im}}\left(x_{2}\right)$, can be expressed by linear transport of an (un-attenuated) field intensity $\mathrm{U}$ at position $Z$,

$$
I_{\mathrm{im}}\left(x_{2}, z\right)=\left[U\left(x_{1}, z\right)\right]^{2} * *\left[h_{z}\left(x_{1}, x_{2}\right)\right]^{2}
$$

where we define $x_{1}$ and $x_{2}$ as two sets of coordinates, corresponding to the planes perpendicular to the optical axis at $z_{1}$ and $z_{2}$ and $* *: \mathbf{R}^{2} \rightarrow \mathbf{R}^{2}$ is the convolution operator in two dimensions. The second assumption is, that the local field propagation within the sample can be done by diffraction-free parallel wave approximation.

For cleaner notation, we construct $h_{z}\left(x_{1}, x_{2}\right)$ so that it includes all linear scaling (magnification, inversion), thus we can express the field transfer simply as a $2 \mathrm{D}$ convolution of the local field plane and a 2D PSF $\left[h_{Z}(x)\right]^{2}$. Using these assumptions Otón et al. [28] construct the derivative of $I_{\mathrm{im}}^{z}$ with respect to $z$ by considering a partially cut sample and adding to it slices of thickness $\Delta z$ (see Fig. A.8).

Following the derivation by Otón et al. [28], we construct the image formation by finite difference of the image, but applying this directly on the logarithm of the normalized image, that is we seek an approximation to the normalized image

$$
f(x, z)=\ln \frac{I_{\mathrm{im}}(x, z)}{I_{\mathrm{im}}(x, z)}:=f(z)
$$

where we drop the explicit notation of $\chi$ for cleaner notation. Constructing the finite difference and substituting for the linear transfer in Eq. (A.1) yields

$$
\ln \frac{I_{\mathrm{im}}(z+\Delta z)}{I_{\mathrm{im}}(z)}=\ln \frac{U(z+\Delta z)^{2} * * h(z+\Delta z)^{2}}{U(z)^{2} * h(z)^{2}}
$$

For the left-hand side, we note that from the series expansion

$$
\ln (x)=(x-1)-\frac{1}{2}(x-1)^{2}+\ldots
$$


we can neglect higher order terms as, $x \approx 1$. For the right-hand side, diffraction-free propagation of the field (to linear accuracy) leads to an intensity of

$$
U(z+\Delta z)^{2} \approx U(z)^{2}-U(z)^{2} \mu(z) \Delta z
$$

and as the PSF can be assumed smooth, we can also assume that

$$
\frac{U(z)^{2} * * h(z+\Delta z)^{2}}{U(z)^{2} * * h(z)^{2}} \approx 1
$$

yielding

$$
\frac{f(z+\Delta z)-f(z)}{\Delta z} \approx-\frac{U(z)^{2} \mu(z)^{*} * h(z+\Delta z)^{2}}{U(z)^{2} *{ }^{2}(z)^{2}} .
$$

To get rid of the final non-linear terms, we assume that $\mathrm{U}(z)^{2}$ is smooth enough within a reasonable extent of $h$, such that this function can be approximated by

$$
\frac{f(z+\Delta z)-f(z)}{\Delta z} \approx-\mu(z) * * h(z+\Delta z)^{2} .
$$

Integrating on both sides of this finite difference approximation of the derivative, we get

$$
\ln \frac{I_{\mathrm{im}}}{I_{\mathrm{im}}^{0}} \approx \int_{\mathrm{R}}-\mu(z) * * h_{z}^{2} \mathrm{~d} z
$$

With suitable discretization, we can now express the right-hand side as a linear projection matrix $\boldsymbol{A}_{h} \boldsymbol{\mu}$, yielding our approximation, Eq. (3).

\section{Appendix A.1. Error with regards to the smoothness of $U(z)$}

To reason about the necessary assumptions needed for Eq. (A.8), let us expand the error at an arbitrary point $x$,

$$
\operatorname{err}(x)=\int \mu(z, t)\left(1-\frac{U(z, t)^{2}}{U(z)^{2} * * h(z)^{2}(x)}\right) \cdot h(z+\Delta z, x-t)^{2} \mathrm{~d} t
$$

This error vanishes at two distinct limits: if $U(z)$ approaches a constant $U$ and if $h(z)^{2}$ approaches a delta peak. For other cases an exact interpretation of the error is nontrivial. Interestingly e.g., as shown in Supplementary materials (Fig. S.7), the optimal PSF for the tomographic inversion may deviate slightly from the PSF used in the forward model. 
To test the assumptions for the forward model in an experimental setting, we calculated the difference on the three forward models of a reconstruction of a biological sample taken with XM2 on beamline 2.1 at the ALS in LBNL [22]. In Fig. A.9 we show the error of a BeerLambert projection and our linear PSF approximation, respectively, as compared to the nonlinear projection model of Eq. (2). It is evident that the error of the linear approximation is acceptable except for at the edges of the samples. This is expected, as the samples in XM2 are mounted in capillary tubes. The capillary tubes produce large gradients in the LAC, where the assumption of a relatively smooth local field fails and the error term in Eq. (A.10) is non-negligible. This kind of error is of course sample dependent, and e.g. dense organelles, fiducial markers and sample holders are all expected to decrease the validity of the model. Nevertheless, the improvement with the model will be still visible

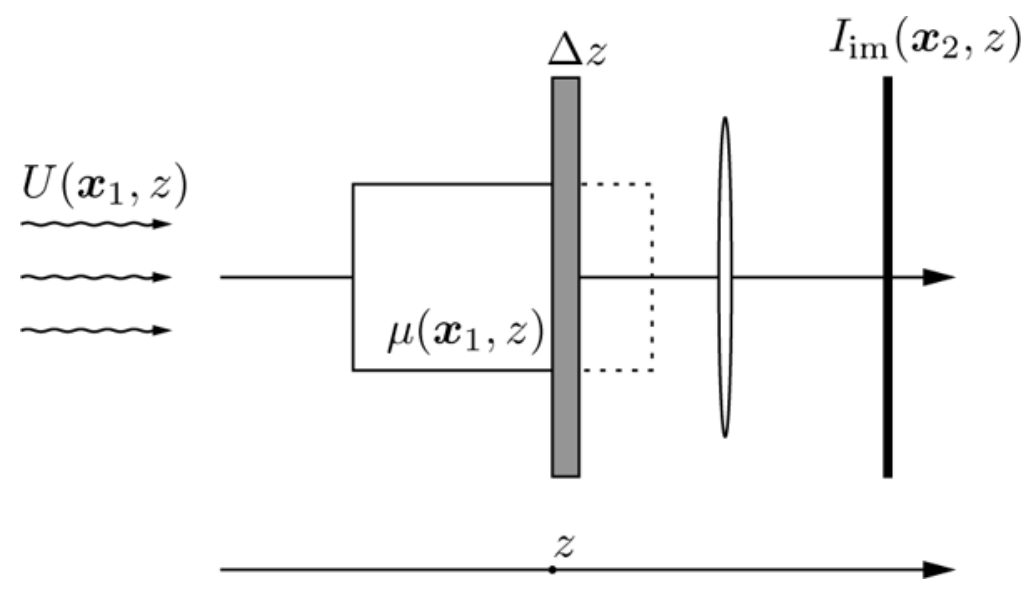

Figure A.8:

The PSF projection is constructed by assuming the image of a "partially cut" sample can be incoherently transferred to the image plane by linear transport. The resulting image $I$ of the whole sample can be constructed by finite difference by adding slices of thickness $\mathrm{d} z$. 

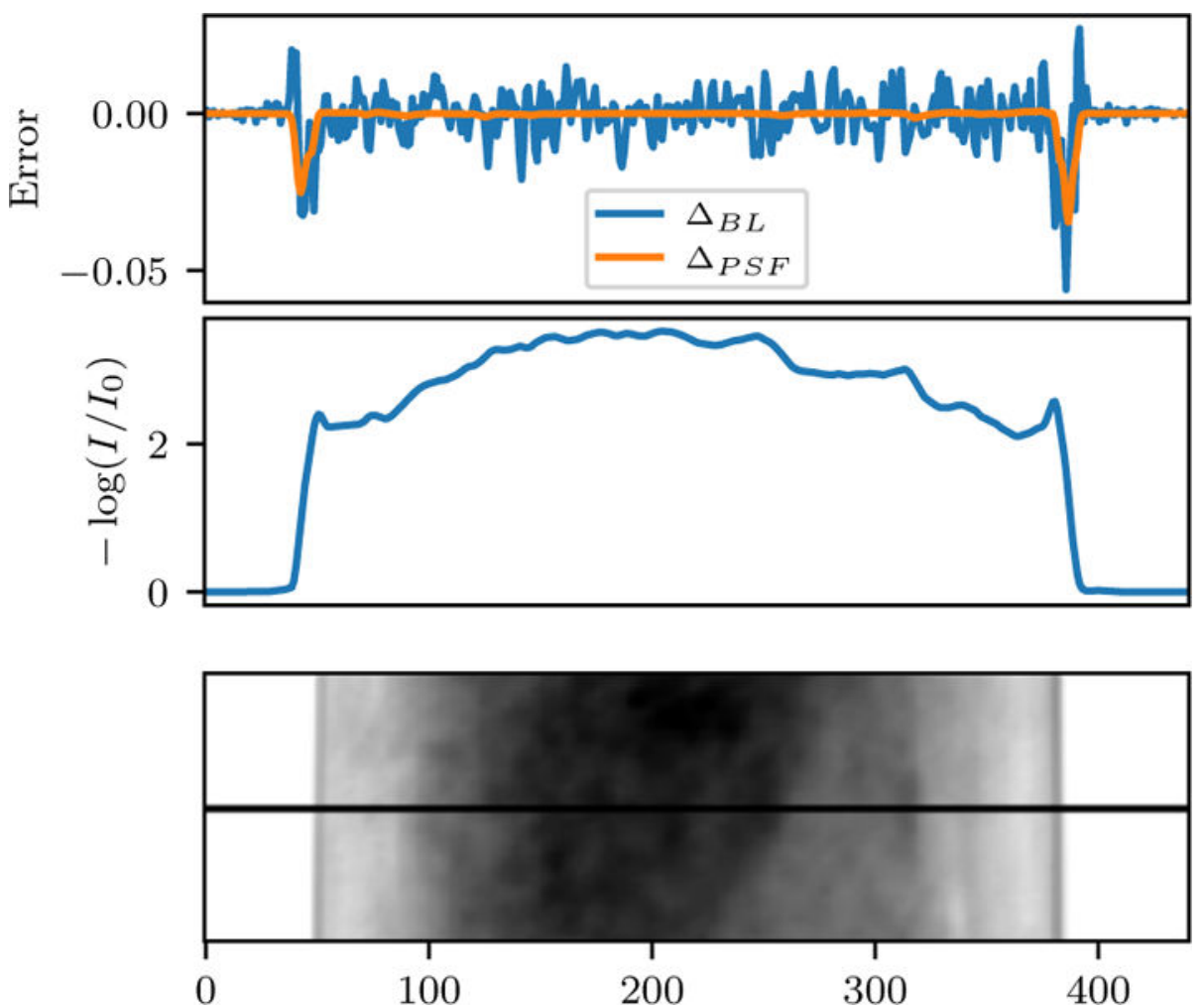

Figure A.9:

The error of the linear model of the projection as compared to the non-linear model of Eq. (2). $\Delta_{B L}$ and $\Delta_{P S F}$ are to the errors of the linear approximation using parallel projection, $\log \left(I / I_{O}\right)+\boldsymbol{A} \boldsymbol{x}$, and PSF projection, $\log \left(I / I_{O}\right)+\boldsymbol{A}_{\boldsymbol{h}} \boldsymbol{x}$, respectively.

\section{References}

[1]. Attwood D (2007). Soft x-rays and extreme ultraviolet radiation: principles and applications. Cambridge University Press.

[2]. Bertilson M, von Hofsten O, Hertz HM, and Vogt U (2011). Numerical model for tomographic image formation in transmission X-ray microscopy. Optics Express 19(12), 11578-11583. [PubMed: 21716389]

[3]. Born M and Wolf E (1970). Principles of Optics: Electromagnetic Theory of Propagation, Interference and Diffraction of Light. Pergamon Press Ltd, Headington Hill Hall, Oxford.

[4]. Carzaniga R, Domart M-C, Collinson LM, and Duke E (2013, 11). Cryo-soft X-ray tomography: a journey into the world of the native-state cell. Protoplasma 251 (2), 449-458. [PubMed: 24264466]

[5]. Chen H-Y, Chiang DM-L, Lin Z-J, Hsieh C-C, Yin G-C, Weng I-C, Guttmann P, Werner S, Henzler K, Schneider G, et al. (2016). Nanoimaging granule dynamics and subcellular structures in activated mast cells using soft x-ray tomography. Scientific reports 6, 34879. [PubMed: 27748356]

[6]. Chiappi M, Conesa JJ, Pereiro E, Sorzano COS, Rodriguez MJ, Henzler K, Schneider G, Chichon FJ, and Carrascosa JL (2016, 3). Cryo-soft X-ray tomography as a quantitative three-dimensional tool to model nanoparticle:cell interaction. Journal of Nanobiotechnology 14 (1).

[7]. Ciompi F, de Hoop B, van Riel SJ, Chung K, Scholten ET, Oudkerk M, de Jong PA, Prokop M, and van Ginneken B (2015). Automatic classification of pulmonary peri-fissural nodules in computed tomography using an ensemble of $2 \mathrm{D}$ views and a convolutional neural network outof-the-box. Medical image analysis 26 (1), 195-202. [PubMed: 26458112] 
[8]. Duke E, DENT K, RAZI M, and COLLINSON LM $(2014,6)$. Biological applications of cryo-soft $\mathrm{X}$-ray tomography. Journal of Microscopy, 65-70.

[9]. Goodman JW (1996). Introduction to Fourier optics (Second ed.). THE McGRAW-HILL COMPANIES, INC New York St Louis San Francisco Auckland Bogot6 Caracas Lisbon London Madrid Mexico City Milan Montreal New Delhi San Juan Singapore Sydney Tokyo Toronto.

[10]. Hansen PC (1998). Rank-deficient and discrete ill-posed problems: numerical aspects of linear inversion, Volume 4 Siam.

[11]. Henke B, Gullikson E, and Davis J (1993, 7). X-Ray Interactions: Photoabsorption, Scattering, Transmission, and Reflection at $\mathrm{E}=50-30,000 \mathrm{eV}, \mathrm{Z}=1-92$. Atomic Data and Nuclear Data Tables 54 (2), 181-342.

[12]. Herman GT (2009). Fundamentals of computerized tomography: image reconstruction from projections. Springer Science \& Business Media.

[13]. Hertz H, von Hofsten O, Bertilson M, Vogt U, Holmberg A, Reinspach J, Martz D, Selin M, Christakou A, Jerlstrom-Hultqvist J, and Svard S (2012, 2). Laboratory cryo soft X-ray microscopy. Journal of Structural Biology 177(2), 267-272. [PubMed: 22119891]

[14]. Hopkins HH $(1953,5)$. On the Diffraction Theory of Optical Images. Proceedings of the Royal Society A: Mathematical, Physical and Engineering Sciences 217(1130), 408-432.

[15]. Jensen GJ and Kornberg RD (2000, 7). Defocus-gradient corrected back-projection. Ultramicroscopy 84 (1-2), 57-64. [PubMed: 10896140]

[16]. Kak AC and Slaney M (1988). Principles of computerized tomographic imaging. IEEE press.

[17]. Kazantsev IG, Klukowska J, Herman GT, and Cernetic L (2010). Fully three-dimensional defocus-gradient corrected backprojection in cryoelectron microscopy. Ultramicroscopy 110 (9), 1128-1142. [PubMed: 20462697]

[18]. Klukowska J and Herman GT (2014). Reconstruction from Microscopic Projections with Defocus-Gradient and Attenuation Effects, pp. 157-186. New York, NY: Springer New York.

[19]. Klukowska J, Herman GT, Oton J, Marabini R, and Carazo J-M $(2014,12)$. The soft X-ray transform. Inverse Problems 30(12), 125015.

[20]. Kohl H and Reimer L (2008). Transmission Electron Microscopy: Physics of Image Formation (5 ed.). 1556-1534. Springer New York.

[21]. Le Gros MA, Clowney EJ, Magklara A, Yen A, Markenscoff-Papadimitriou E, Colquitt B, Myllys M, Kellis M, Lomvardas S, and Larabell CA (2016). Soft x-ray tomography reveals gradual chromatin compaction and reorganization during neurogenesis in vivo. Cell reports 17(8), 2125-2136. [PubMed: 27851973]

[22]. Le Gros MA, McDermott G, Cinquin BP, Smith EA, Do M, Chao WL, Naulleau PP, and Larabell CA (2014). Biological soft X-ray tomography on beamline 2.1 at the Advanced Light Source. Journal of synchrotron radiation 21 (6), 1370-1377. [PubMed: 25343808]

[23]. Li F, Guan Y, Xiong Y, Zhang X, Liu G, and Tian Y (2017). Method for extending the depth of focus in X-ray microscopy. Optics Express 25 (7), 7657-7667. [PubMed: 28380885]

[24]. McNally JG, Rehbein S, Pratsch C, Werner S, Schneider G, et al. (2016). 3D PSF Measurement for a Soft X-ray Microscope and Comparison to Theory In Computational Optical Sensing and Imaging. Optical Society of America.

[25]. Natterer F (1986). Computerized Tomography, pp. 1-8. Wiesbaden: Vieweg+Teubner Verlag.

[26]. Oton J, Pereiro E, Conesa JJ, Chichón FJ, Luque D, Rodríguez JM, Perez-Berna AJ, Sorzano COS, Klukowska J, Herman GT, et al. (2017). Xtend: Extending the depth of field in cryo soft xray tomography. Scientific Reports 7.

[27]. Otón J, Pereiro E, Perez-Bernó AJ, Millach L, Sorzano COS, Marabini R, and Carazo JM (2016). Characterization of transfer function, resolution and depth of field of a soft x-ray microscope applied to tomography enhancement by wiener deconvolution. Biomedical Optics Express 7(12), 5092-5103. [PubMed: 28018727]

[28]. Otón J, Sorzano C, Pereiro E, Cuenca-Alba J, Navarro R, Carazo JM, and Marabini R (2012, 4). Image formation in cellular X-ray microscopy. Journal of Structural Biology 178(1), 29-37. [PubMed: 22343468] 
[29]. Otón J, Sorzano COS, Chichón FJ, Carrascosa JL, Carazo JM, and Marabini R (2014). Soft XRay Tomography Imaging for Biological Samples, pp. 187-220. New York, NY: Springer New York.

[30]. Otón J, Sorzano COS, Marabini R, Pereiro E, and Carazo JM (2015, 4). Measurement of the modulation transfer function of an $\mathrm{x}$-ray microscope based on multiple fourier orders analysis of a siemens star. Opt. Express 23(8), 9567-9572. [PubMed: 25968993]

[31]. Parkinson DY, Epperly LR, McDermott G, Le Gros MA, Boudreau RM, and Larabell CA (2013). Nanoimaging cells using soft X-ray tomography. Nanoimaging: Methods and Protocols, 457481.

[32]. Parkinson DY, Knoechel C, Yang C, Larabell CA, and Le Gros MA (2012). Automatic alignment and reconstruction of images for soft X-ray tomography. Journal of structural biology 177(2), 259-266. [PubMed: 22155289]

[33]. Patwardhan A, Ashton A, Brandt R, Butcher S, Carzaniga R, Chiu W, Collinson L, Doux P, Duke E, Ellisman MH, Franken E, Grünewald K, Heriche J-K, Koster A, Kühlbrandt W, Lagerstedt I, Larabell C, Lawson CL, Saibil HR, Sanz-García E, Subramaniam S, Verkade P, Swedlow JR, and Kleywegt GJ $(2014,10)$. A 3D cellular context for the macromolecular world. Nature Structural \& Molecular Biology 21 (10), 841-845.

[34]. Pelt DM and Sethian JA (2018). A mixed-scale dense convolutional neural network for image analysis. Proceedings of the National Academy of Sciences 115(2), 254-259.

[35]. Radon J (1917). On determination of functions by their integral values along certain multiplicities. Berichte der Sächische Akademie der Wissenschaften Leipzig, (Germany) 69, 262-277.

[36]. Roth HR, Lu L, Seff A, Cherry KM, Hoffman J, Wang S, Liu J, Turkbey E, and Summers RM (2014). A new 2.5 D representation for lymph node detection using random sets of deep convolutional neural network observations. In International Conference on Medical Image Computing and Computer-Assisted Intervention, pp. 520-527. Springer.

[37]. Schneider G, Guttmann P, Heim S, Rehbein S, Mueller F, Nagashima K, Heymann JB, Muüller WG, and McNally JG $(2010,11)$. Three-dimensional cellular ultrastructure resolved by X-ray microscopy. Nature Methods 7(12), 985-987. [PubMed: 21076419]

[38]. Selin M, Fogelqvist E, Holmberg A, Guttmann P, Vogt U, and Hertz HM (2014). 3D simulation of the image formation in soft x-ray microscopes. Optics Express 22(25), 30756-30768. [PubMed: 25607024]

[39]. Selin M, Fogelqvist E, Werner S, and Hertz HM (2015). Tomographic reconstruction in soft x-ray microscopy using focus-stack back-projection. Optics letters 40(10), 2201-2204. [PubMed: 26393699]

[40]. Sheppard C and Mao X (1989). Three-dimensional imaging in a microscope. JOSA A 6(9), 1260-1269.

[41]. Shkolyar A, Gefen A, Benayahu D, and Greenspan H (2015). Automatic detection of cell divisions (mitosis) in live-imaging microscopy images using Convolutional Neural Networks. In 2015 37th Annual International Conference of the IEEE Engineering in Medicine and Biology Society (EMBC), pp. 743-746. IEEE.

[42]. Streibl N (1985, 2). Three-dimensional imaging by a microscope. Journal of the Optical Society of America A 2(2), 121.

[43]. Uchida M, Sun Y, McDermott G, Knoechel C, Gros MAL, Parkinson D, Drubin DG, and Larabell CA $(2010,12)$. Quantitative analysis of yeast internal architecture using soft X-ray tomography. Yeast 28(3), 227-236. [PubMed: 21360734]

[44]. van Kempen GM, van der Voort H. T., Bauman JG, and Strasters KC (1996). Comparing maximum likelihood estimation and constrained tikhonov-miller restoration. IEEE Engineering in Medicine and Biology Magazine 15(1), 76-83.

[45]. von Hofsten O, Takman PA, and Vogt U (2007, 8). Simulation of partially coherent image formation in a compact soft X-ray microscope. Ultramicroscopy 107(8), 604-609. [PubMed: 17261350]

[46]. Voortman LM, Franken EM, van Vliet LJ, and Rieger B (2012). Fast, spatially varying ctf correction in tem. Ultramicroscopy 118, 26-34. [PubMed: 22728402] 
[47]. Voortman LM, Stallinga S, Schoenmakers RH, van Vliet LJ, and Rieger B (2011, 7). A fast algorithm for computing and correcting the CTF for tilted, thick specimens in TEM. Ultramicroscopy 111 (8), 1029-1036. [PubMed: 21740865]

[48]. Weiß D, Schneider G, Niemann B, Guttmann P, Rudolph D, and Schmahl G (2000). Computed tomography of cryogenic biological specimens based on X-ray microscopic images. Ultramicroscopy 84 (3-4), 185-197. [PubMed: 10945329]

[49]. Yoo H, Song I, and GWEON D-G (2006). Measurement and restoration of the point spread function of fluorescence confocal microscopy. Journal of microscopy 221 (3), 172-176. [PubMed: 16551278]

[50]. Zhang W, Li R, Deng H, Wang L, Lin W, Ji S, and Shen D (2015). Deep convolutional neural networks for multi-modality isointense infant brain image segmentation. Neuroimage 108, 214 224. [PubMed: 25562829] 


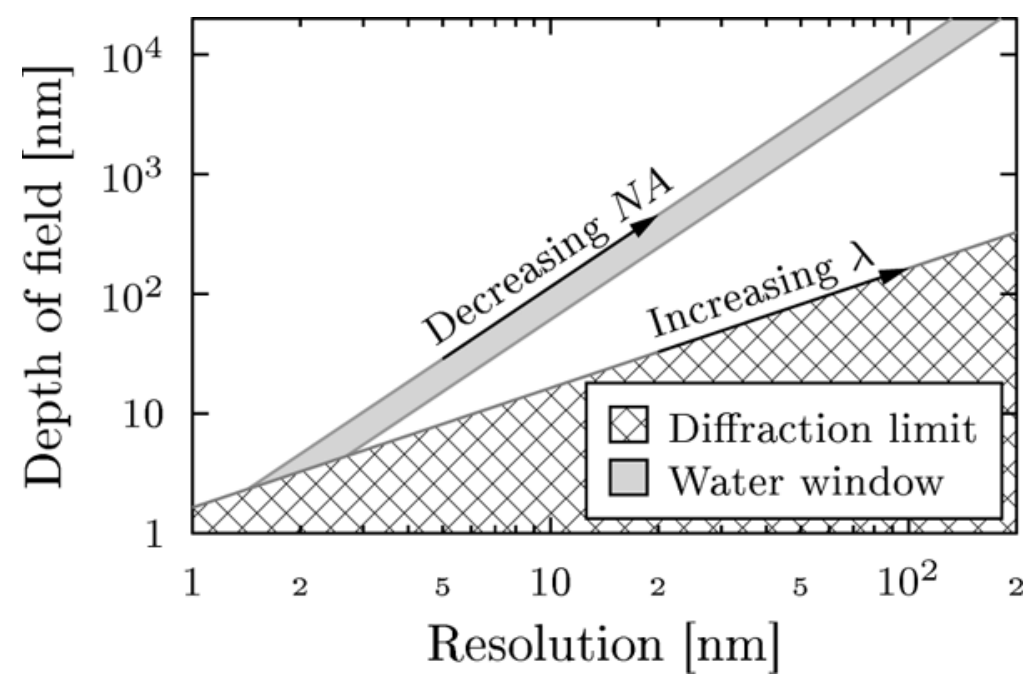

Figure 1:

The relation between resolution and the depth of field for an ideal lens and monochromatic light. The diffraction limit shown here is defined as $r=0.61 \lambda / N A$ for $N A=1$. The water window shows accessible resolution and depth of field for soft $\mathrm{x}$-ray energy range. 


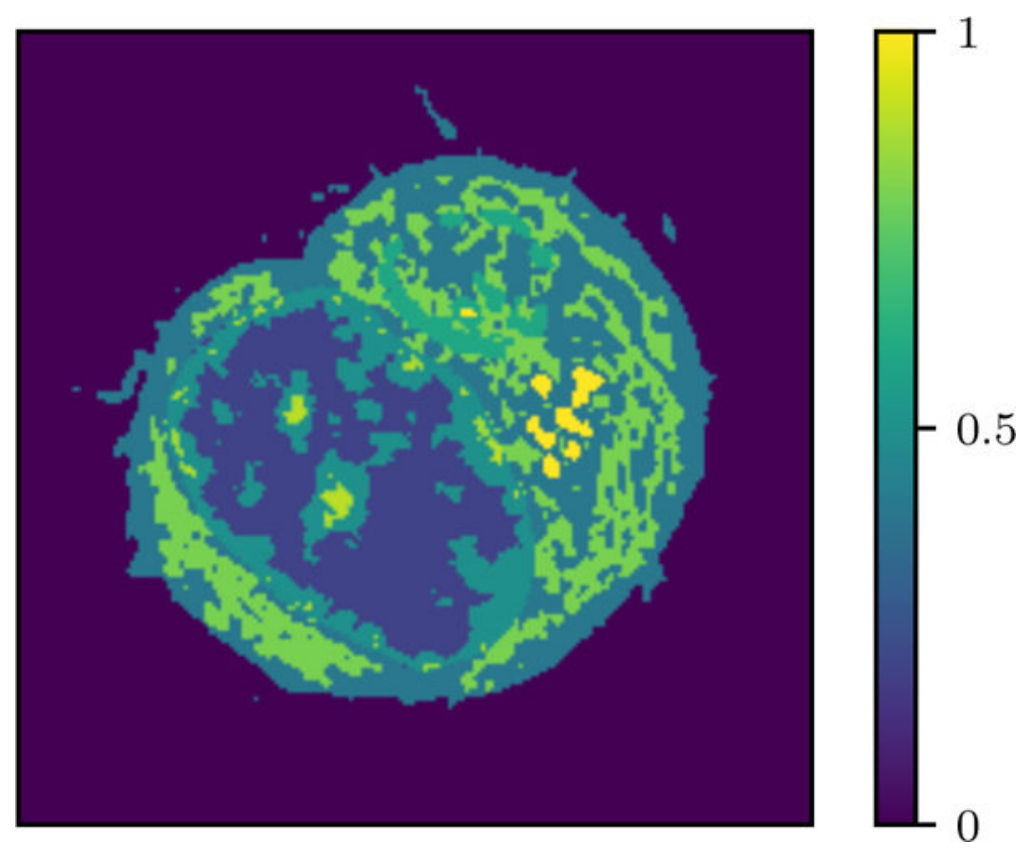

Figure 2:

The binary phantom of a human B cell used for the numerical calculations. The colormap shown here is scaled with the maximum LAC value of the phantom. 

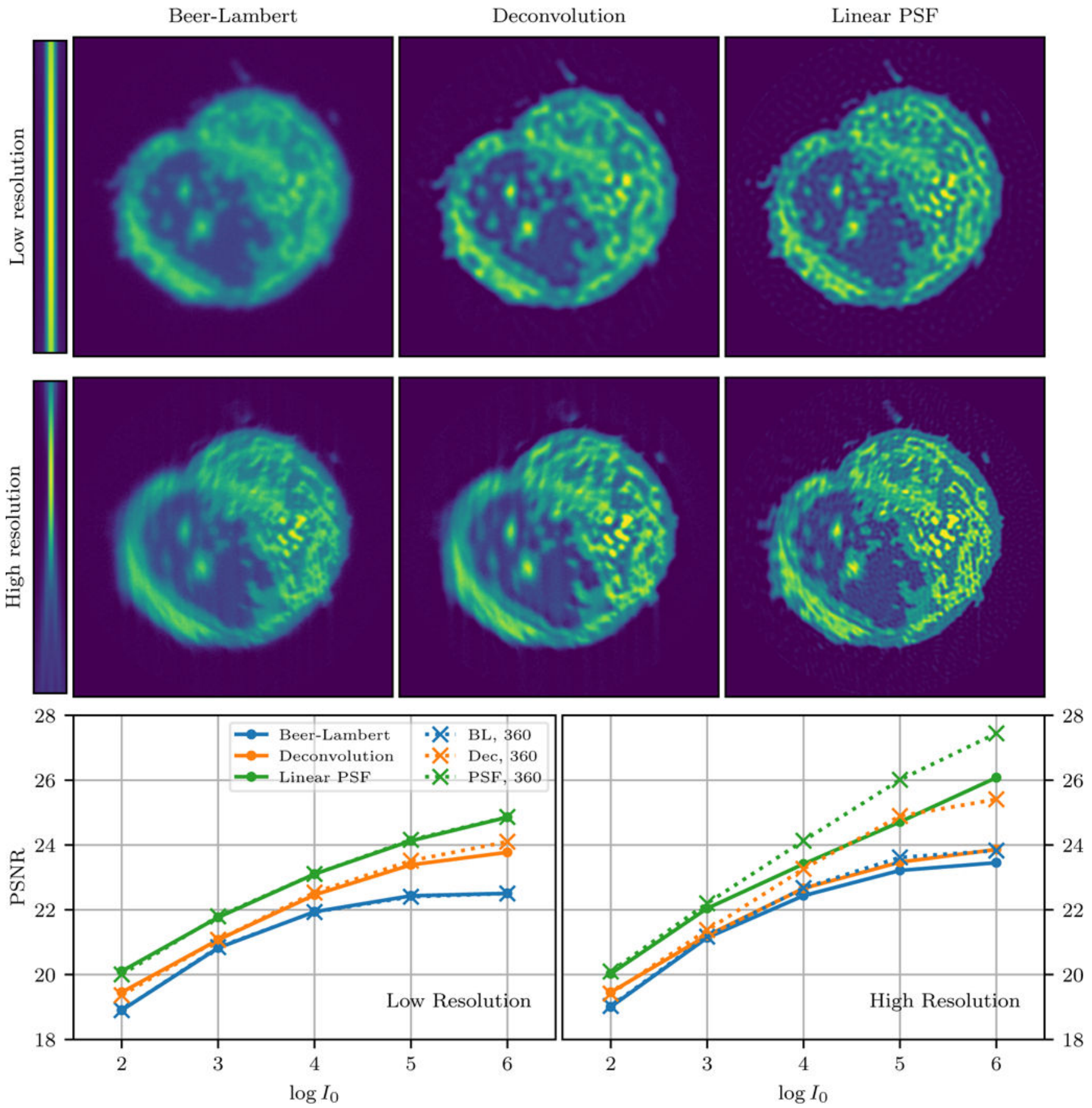

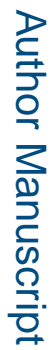

Figure 3:

Reconstructions using Beer-Lambert approximation (left) global deconvolution (middle) and PSF projection (right) for the two PSF kernels considered shown on the far left. Here the images were relatively noiseless with $I_{0}=10^{6}$. The contrast of the image intensity is the same as in the reference figure Fig. 2. The bottom row shows the PSNR (Eq. 5) for the reconstructions of the numerical phantom sample as a function of intensity. The solid and dotted lines show $180^{\circ}$ and $360^{\circ}$ rotation acquisition strategies, respectively. 

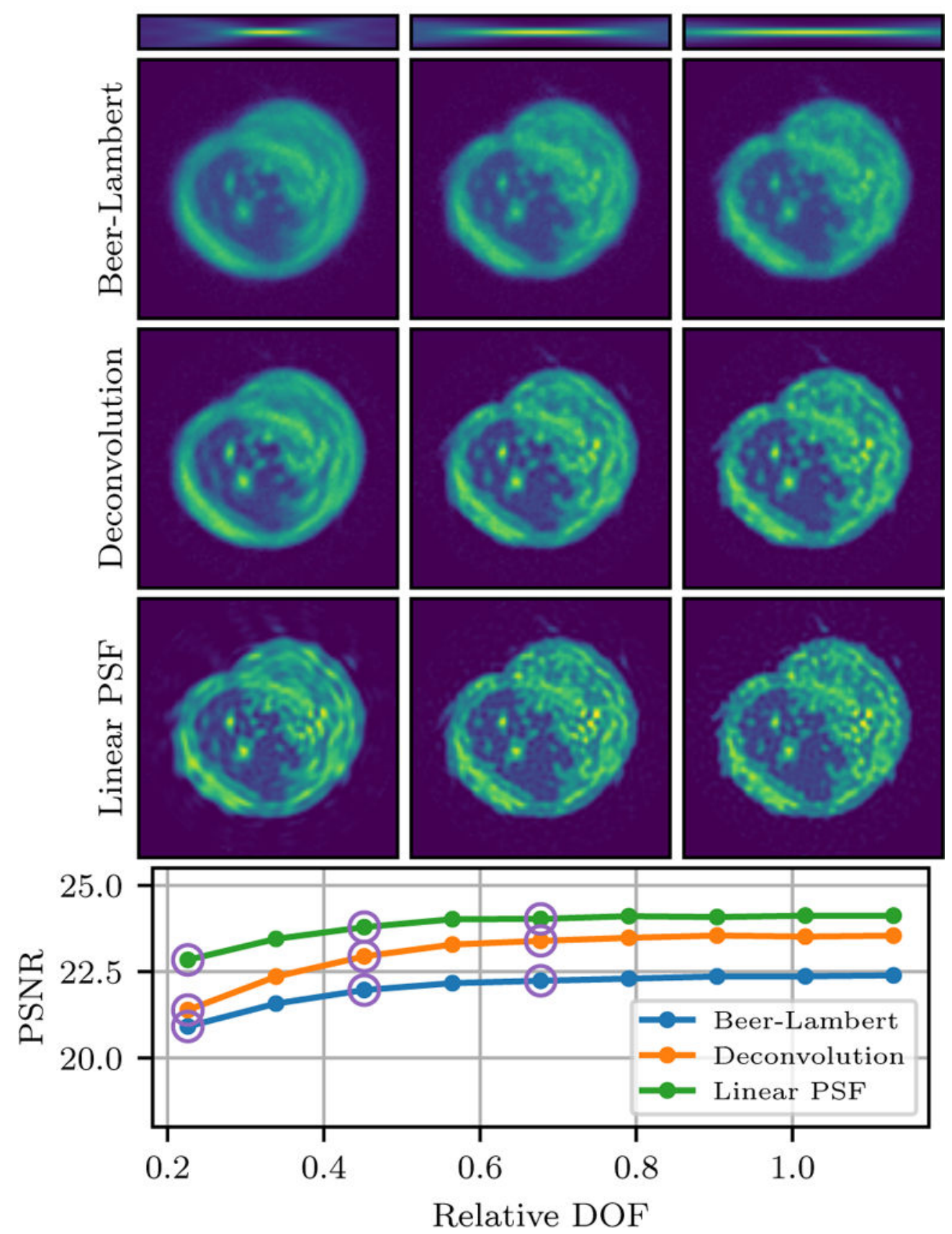

Figure 4:

Three selected examples of the effect of a limited DOF $(23 \%, 45 \%$ and $68 \%$ of the specimen) on the reconstruction quality of the phantom with the corresponding PSNR for the reconstructions (shown below). The shown reconstructions and corresponding PSNR are highlighted by additional circular markers. Projections were made with $I_{0}=10^{5}$. 


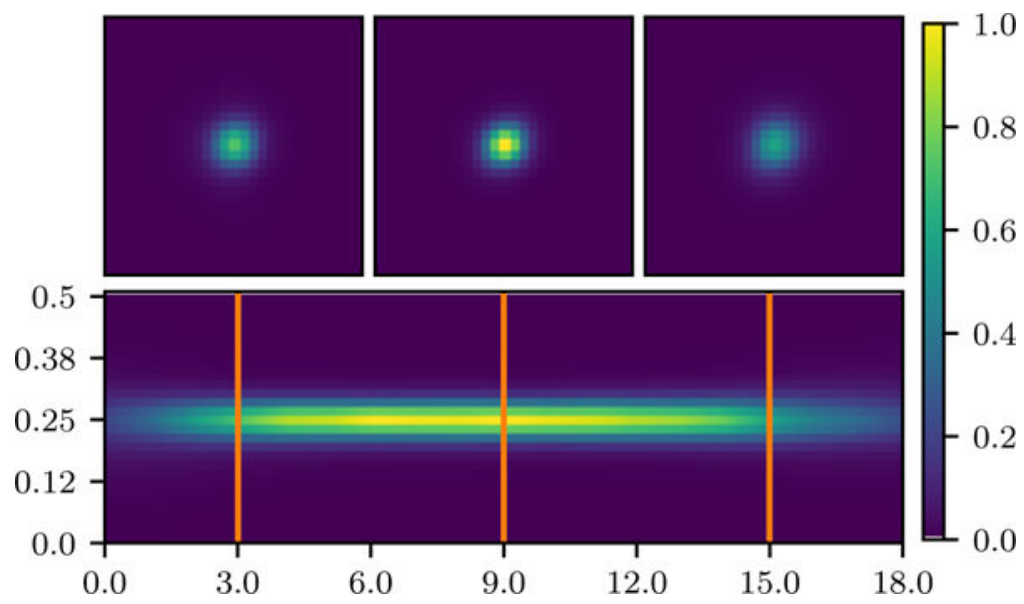

Figure 5:

The sagittal slice (along $\mathrm{x}$-ray propagation) of the extracted PSF and transverse slices ( 0.5 $\mu \mathrm{m} \cdot 0.5 \mu \mathrm{m}$ ) at positions with respect to the focus. The length of the PSF shown here is 18 $\mu \mathrm{m}$. The shown image intensity is scaled with the maximal value of the PSF. 

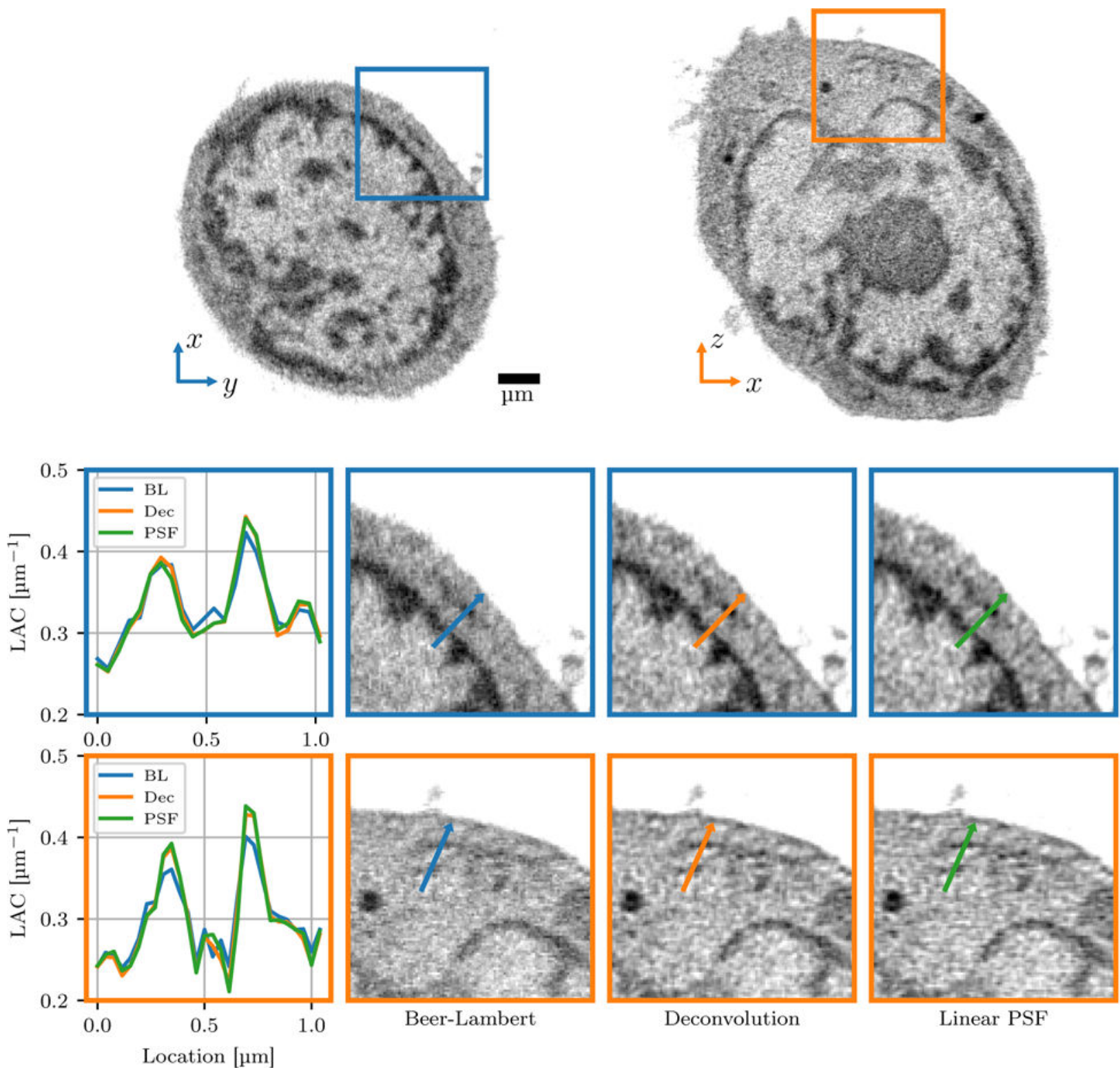

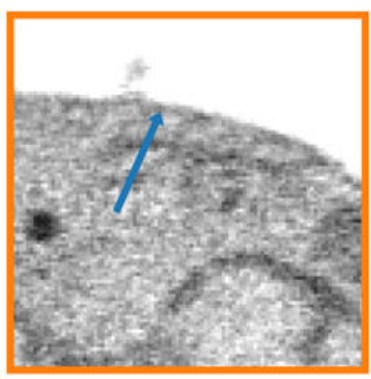

Beer-Lambert

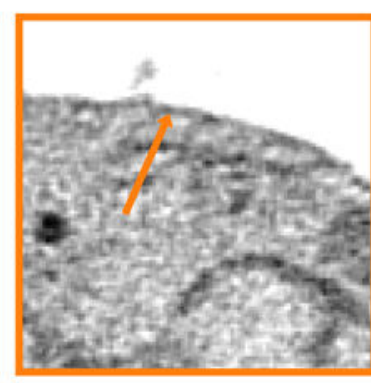

Deconvolution

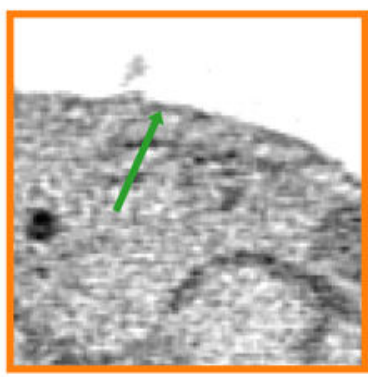

Linear PSF

Figure 6:

An in-plane slice (plane orthogonal to the axis of rotation) and a transverse plane of the reconstruction of a mouse B-cell. The two rows show regions of interest $(3.5 \mu \mathrm{m} \cdot 3.5 \mu \mathrm{m})$ from the corresponding slice. The arrows correspond to line profile depicted in the corresponding leftmost plot, which shows the LAC profile along the line. 
lac

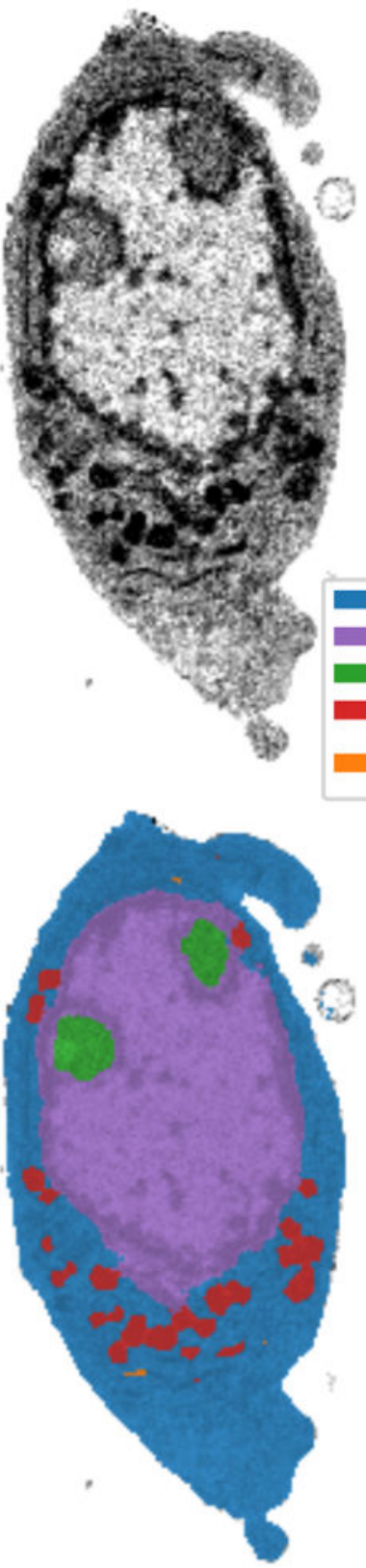

Beer-Lambert

Manual

Cell

Nucleus

Nucleoli

Mitochondria

Endoplasmic reticulum

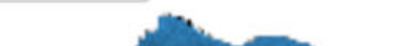

Figure 7:

Example of a trained multi-class labeling using the algorithm presented by Pelt and Sethian [34]. The results are presented in Table 1. 
Table 1:

Precision and recall of the multi-label classification. Nucleus, nucleoli, mitochondria (Mit.), endoplasmic reticulum and cell material.

\begin{tabular}{|c|c|c|c|c|c|c|}
\hline & & Cell & Nucleus & Nucleoli & Mit. & ER \\
\hline \multirow{2}{*}{ Precision } & $\mathrm{BL}$ & 0.952 & 0.915 & 0.968 & 0.845 & 0.309 \\
\hline & PSF & 0.943 & 0.927 & 0.973 & 0.924 & 0.511 \\
\hline \multirow{2}{*}{ Recall } & $\mathrm{BL}$ & 0.896 & 0.981 & 0.806 & 0.838 & 0.070 \\
\hline & PSF & 0.913 & 0.990 & 0.852 & 0.798 & 0.381 \\
\hline
\end{tabular}

